\title{
MESH DISPERSION MINIMIZATION ALGORITHMS WITHIN EXPLICIT FINITE-DIFFERENCE SCHEMES TO CALCULATE TRANSIENT WAVE PROCESSES IN ELASTIC MEDIA AND COMPOSITE STRUCTURES
}

\author{
SAGDULLA ABDUKADIROV ${ }^{1,2}$ \\ ${ }^{1}$ Tashkent Institute of Architecture and Civil Engineering, Uzbekistan \\ ${ }^{2}$ Tashkent Institute of Irrigation and Agricultural Mechanization Engineers, Uzbekistan
}

\begin{abstract}
Precise calculation of wave fronts and high-gradient components is always of utmost importance for problems of numerical simulation of wave processes in media and composite structures. The usage mesh algorithms come across specific obstacles, which do not allow to accurately calculate such disturbances localized at the loading area or propagated with time. One of such obstacles (notably in the problems with singularities) is the spurious effect caused by the mesh dispersion responsible for the emergence of high-frequency "parasite" oscillations damaged the computer solution. In this work, advanced numerical algorithms within the explicit finite-difference scheme are developed exactly for very purpose - to precisely calculate wave processes with singularities. The algorithms are constructed with the condition that dependence domains are the same (or maximally closed) in differential and difference equations corresponding to continual and discrete models, respectively. In the designed algorithms, the influence of spurious effects of numerical dispersion is suppressed (or essentially minimized) that allows discontinuities in fronts and high-gradient components to be accurately calculated. A set of examples of computer simulations of linear and nonlinear wave processes are presented. Among them are (a) impact propagation in a waveguide resting on an elastic foundation, (b) cylindrical and spherical waves, and (c) wave propagation and fracture pattern in a unidirectional composite. Comparison of results calculated by conventional and developed algorithms clearly shows the advantage of the latter. To this end, precise numerical solutions (in mesh points of the discrete space) are obtained for the problems listed above.
\end{abstract}

Keywords: transient wave dynamics, explicit finite difference scheme, mesh dispersion, fracture of unidirectional composites.

\section{INTRODUCTION}

Significant rise in influence of the microstructure on the wave pattern essentially restricts capabilities of analytical modeling. On the other hand, numerical solutions allow to obtain quantitative evaluations of the process under study and to explain physical consequences. At the same time, mesh algorithms used in computer codes come across specific obstacles, which do not allow to calculate accurately wave fronts and high-gradient components localized at the loading area or propagated with time. One of such obstacles is the spurious effect caused by the Mesh Dispersion (MD) and responsible for the emergence of the highfrequency "parasite" noise damaged the computer solution. This phenomenon manifested notably in the problems with singularities and multiple reflections, possesses own highfrequency patterns which are typical for compound media and composites. The studies of the $M D$ in initial-boundary problems have a long-standing history and extensive literature beginning with classical works [1]-[4]. The present study is the further development of the so-called Mesh Dispersion Minimization $(M D M)$ procedure in the explicit finite difference algorithms originally presented in [5] and then used in calculations of diverse range of practical engineering problems in [6]-[11]. 
The $M D M$ technique is based on a generalized concept of the Courant condition linked temporal and spatial mesh steps with the wave velocity, which reflects properties of the material at hand. An important technical advantage of $M D M$ is that it utilizes the same homogeneous mesh for both high-gradient and smoothed wave components.

The $M D M$ algorithm for dispersionless waveguides proves to be stable at the Courant number $\lambda=c_{0} \Delta t / \Delta \mathrm{x}=1$ [5], where $\Delta t$ and $\Delta x$ are the time and spatial dimensions of the difference mesh, $c_{0}$ is the sound velocity. The equality $\lambda=1$ has the simple physical sense: during one step in time, the wave passes one spatial step. Here the solutions of continual and difference problems coincide in mesh points.

The algorithms controlling $M D$ have been also developed in many contemporary studies, see for example, [12]-[17] intended for a wide spectrum of physical problems; among them the application to the analysis of even financial and stock exchange processes is found (see [12]). Unfortunately, one of important points of $M D$ suppression - the accuracy of computation of wave processes possessing front gaps is not still completely revealed.

In this work, we have built algorithms suppressed $M D$ in linear and nonlinear dispersive waveguides (Section 2). In Section 3, cylindrical and spherical waves possessing fronts are precisely calculated. Finally, in Section 4, we have presented $M D M$ algorithms and examples of computer simulations of the multi-front wave pattern and development of the dynamic fracture realized in a unidirectional composite plate.

\section{ELASTIC WAVEGUIDES}

\subsection{Classical dispersionless waveguide}

Consider the wave propagation problem in a semi-infinite dispersionless waveguide $(x \geq 0)$ subjected by the tension force $F$ at free end $x=0$. We have used the model:

$$
\ddot{u}=c_{0}^{2} u^{\prime \prime}, u^{\prime}(0, t)=-F(t), u(x, 0)=\dot{u}(x, 0)=0,
$$

where $u=u(x, t)$ is displacement, $c_{0}$ is the sound speed, while parameters of the waveguide serves as measurement units. The goal is to choose the most effective calculation scheme.

This simple model (1) is very convenient for demonstration of the main MDM principle.

First of all, we apply the Fourier expansion $u=U \exp [\imath q(c t \pm x)]$ to eqn (1) and obtain the dispersion relation $c=c_{0}$ inherent to the dispersionless free wave propagation in the infinite waveguide $(|x|<\infty)$. Above indicated: $U \sim$ const, $l$ is the imaginary unit, $q$ is the wave number (then $l=2 \pi / q$ is the wavelength), $c$ is the phase velocity.

The explicit "cross" type finite-difference analog of eqn (1) is written as follows:

$$
u_{i}^{k+1}=2 u_{i}^{k}-u_{i}^{k-1}+\lambda^{2}\left(u_{i+1}^{k}-2 u_{i}^{k}+u_{i-1}^{k}\right), \lambda=c_{0} \Delta t / \Delta x
$$

where $x=i \Delta x(i=0, \pm 1, \pm 2, \ldots),, t=k \Delta t(k=0,1,2, \ldots), i$ and $k$ are coordinates of the mesh nodes, $\lambda$ is the Courant number. 
Substituting Fourier expansion $u_{i}^{k}=U \exp [\imath q(c k \Delta t \pm i \Delta x)]$ into eqn (2), we have obtained the following dispersion relation in form $c=c(q)$ : $c=(2 / q \Delta t) \arcsin [\lambda \cdot \sin (q \Delta x / 2)]$ proved the dependence of the phase velocity on the wavenumber $q$ and mesh steps that, generally speaking, determines the wave dispersion in model (2).

At the same time, however, one can detect that in the case $\lambda=1$ this dispersion relation becomes the same that in the continual case: $c=c_{0}$, and the $M D$ is completely eliminated.

In a general case, we have no closed analytical solution of eqn (2) like that for eqn (1) with added initial and boundary conditions, but in case $\lambda=1$ such a solution is obtained with using the mathematical induction technique (see [5]).

Let us compare solutions of eqns (1) and (2) for two versions of boundary loadings at the end $x=0$ : (a) the Heaviside step tension $F(t)=-H(t)$ and (b) the Dirac pulse $F(t)=-\delta(t) ; c_{0}$ serves as measurement unit:

$$
\begin{aligned}
& \text { eqn (1): (a) } u^{\prime}(x, t)=-H(t-x) ; \quad(b) u^{\prime}(x, t)=-1(x=t), u^{\prime}(x, t)=0(x \neq t), \\
& \text { eqn (2): (a) } u^{\prime}(x, t)=-H(k-i) ; \quad(b) u^{\prime}(x, t)=-1(k=i), u^{\prime}(x, t)=0(k \neq i) .
\end{aligned}
$$

(for eqn (1), the well-known d'Alambert solution is used). These solutions coincide in mesh nodes. Such a computer solution is defined as "the accurate numerical solution" (ANS). Snapshots of strains $u^{\prime}$ at $t=100$ are shown in Fig. 1. Note, computer results obtained with $\lambda$ $<1$ are essentially distorted by the $M D$ action.
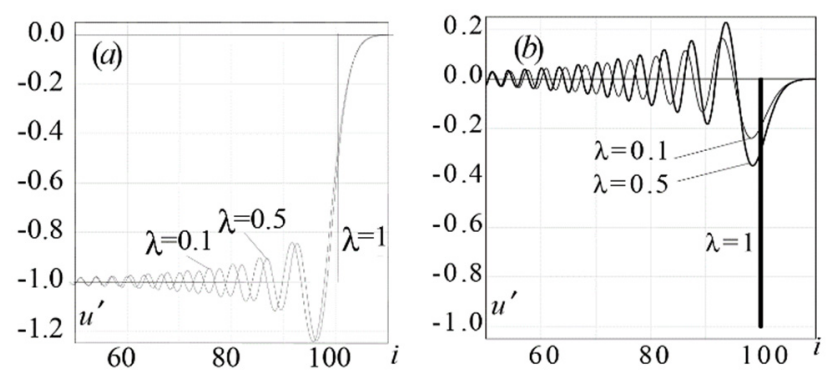

Figure 1: Snapshots of strains at $t=100$ for two kinds of loadings. (a)The Heaviside step; and (b) The Dirac pulse. Oscillating curves are numerical solutions at $\lambda<1$.

\subsection{Dispersive waveguides}

Consider now wave equation

$$
\ddot{u}-c_{0}^{2} u^{\prime \prime}+G(u)=0
$$

known as the generalized Klein-Gordon equation, where $G(u)$ is a finite function. Eqn (4) is widely used in diverse models of physics and structure dynamics.

First, consider linear function $G(u) \equiv g u, g \sim$ const. Then eqn (4) will be written as 


$$
\ddot{u}-c_{0}^{2} u^{\prime \prime}+g u=0
$$

and its dispersion equation is

$$
c(q)=\sqrt{1+g / q^{2}}
$$

which, in contrast with relation $c=q$ corresponding to eqn (1) shows the wave dispersion.

The explicit "cross"-type finite-difference analog of eqn (1) is

$$
u_{i}^{k+1}=2 u_{i}^{k}-u_{i}^{k-1}+\lambda^{2}\left(u_{i+1}^{k}-2 u_{i}^{k}+u_{i-1}^{k}\right)+(\Delta t)^{2} g u_{i}^{k} \quad(\lambda=\Delta t / \Delta x)
$$

where $\Delta x$ and $c_{0}$ are taken as measurement units: $\Delta x=c_{0}=1$, that results in equality $\lambda=\Delta t$. The dispersion relation for eqn (7) is obtained as

$$
c= \pm(2 / q \Delta t) \arcsin \left(\Delta t \sqrt{\sin ^{2}(q / 2)+g / 4}\right)
$$

that proves presence of the $M D$ inevitably distorted the computer solution.

Our aim is to construct such a difference scheme for the discrete analog of eqn (5) so that its dispersion relation would be as close as possible to relation (6). The MDM approach thelps to achieve this goal. We have changed the ordinary local approximation $u=u_{i}^{k}$ in term $g u$ of eqn (7) to a special non-local three-point approximation:

$$
u \sim\left(u_{i+1}^{k}+2 u_{i}^{k}+u_{i-1}^{k}\right) / 4 .
$$

Then, the conventional algorithm (7) is turned to be written as the following:

$$
u_{i}^{k+1}=2 u_{i}^{k}-u_{i}^{k-1}+\lambda^{2}\left[\left(u_{i+1}^{k}-2 u_{i}^{k}+u_{i-1}^{k}\right)+g\left(u_{i+1}^{k}+2 u_{i}^{k}+u_{i-1}^{k}\right) / 4\right] .
$$

(there can be readily shown that approximation orders of eqns (7) and (10) are the same $-(\Delta x)^{2}+(\Delta t)^{2}$, while the dispersion relation for eqn (10) acquires the following form:

$$
c= \pm(2 / q \Delta t) \arcsin \left[\Delta t \sqrt{\sin ^{2}(q / 2)+(g / 4) \cos ^{2}(q / 2)}\right] .
$$

If we set $\lambda=1$ (then $\Delta t=1$ due to the fact that $\Delta x=c_{0}=1$ ) in eqn (10), then extremely short waves of length $l=2(q=\pi)$ will propagate with the same phase velocity $c=1$ as infinitely short waves, $l \rightarrow 0(q \rightarrow \infty)$, in the continual model. As in the free waveguide above, $M D$ is completely eliminated over the entire discrete spectrum. 

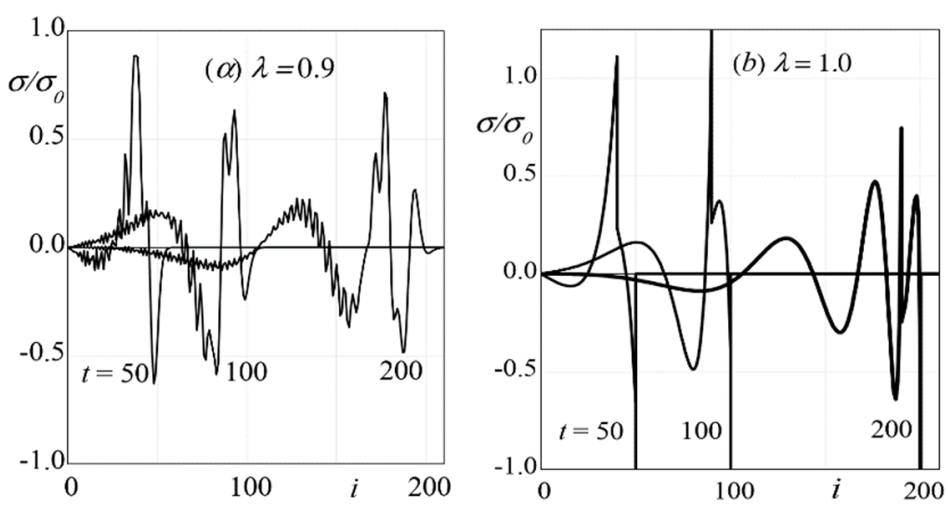

Figure 2: Computer solutions for normalized stresses propagated along the waveguide $(g=0.01)$ under action of pulse of duration $t_{0}=10$. (a) Conventional algorithm (7) with $\lambda=0.9$. (b) MDM algorithm (10) with $\lambda=1$ resulting in the ANS.

In Fig. 2, results of computation of wave propagation processes are compared. We have added zero initial conditions to eqn (10) and the boundary condition as action of the stepwise compression stress at the end $x=0: \sigma(0, t)=-\sigma_{0} H\left(t-t_{0}\right)$, where $\sigma_{0}$ and $t_{0}$ are the pulse amplitude and duration.

The essential distortions are detected of the solution obtained by the conventional algorithm (7) with $\lambda=0.9$, while calculations with the $M D M$ algorithm (10) with $\lambda=1$ can be considered as the $A M S$ : spurious oscillations are absent, and front gaps are clearly detected.

Calculation results below are obtained with $M D M$ algorithms.

The introduced above three-point approximation (9) within $M D M$-algorithms used also in the case of the inhomogeneous foundation: $G(u)=g(x) u(x)$. Although the dispersion equation is absent here, using the so-called method of frozen coefficients helps achieve our goal: we denote $g=\max |g(x)|_{x}$ and change variables in eqn (4): $\bar{x}=x \sqrt{g}, \bar{t}=t \sqrt{g}$. After that $M D M$ algorithm (10) is launched.

Consider now longitudinal wave propagation processes in a semi-infinite waveguide $(x \geq 0)$ resting upon a nonlinear foundation. Let boundary and initial conditions be the same that were used above. In the example below we consider the following initial-boundary problems:

$$
\ddot{u}-c_{0}^{2} u^{\prime \prime}+g u\left(1+g_{0} u^{2}\right)=0, \varepsilon(0, t) \equiv u^{\prime}(0, t)=-F(t), u(x, 0)=\dot{u}(x, 0)=0 .
$$

Our aim is to design the $M D M$ algorithm to problem (12) and to reveal by computer simulations the influence of nonlinearity on the wave propagation process.

Completed tests show that the $M D M$ approximation (9) together with condition $\lambda=1$ results in the dispersionless algorithm

$$
u_{i}^{k+1}=2 u_{i}^{k}-u_{i}^{k-1}+\lambda^{2}\left[\left(u_{i+1}^{k}-2 u_{i}^{k}+u_{i-1}^{k}\right)+g U\left(1+g_{0} U^{2}\right)\right], \quad U=\left(u_{i+1}^{k}+2 u_{i}^{k}+u_{i-1}^{k}\right) / 4,
$$


allowing the problem on the same mesh and with the same accuracy to be calculated as in the linear case.

In Fig. 3, snapshots of linear and nonlinear stress wave patterns are compared at $t=250$. Note, the fundamental difference in front zone is not found in linear and nonlinear solutions (despite the relatively huge value of $g_{0}$ ). This surprising (at first sight) result can be explained by the fact that the package of high frequency oscillations generating in the front zone, propagates together with the front, while the perturbations related to the presence of the foundation (and, in this way, the nonlinearity) moves behind the front zone.

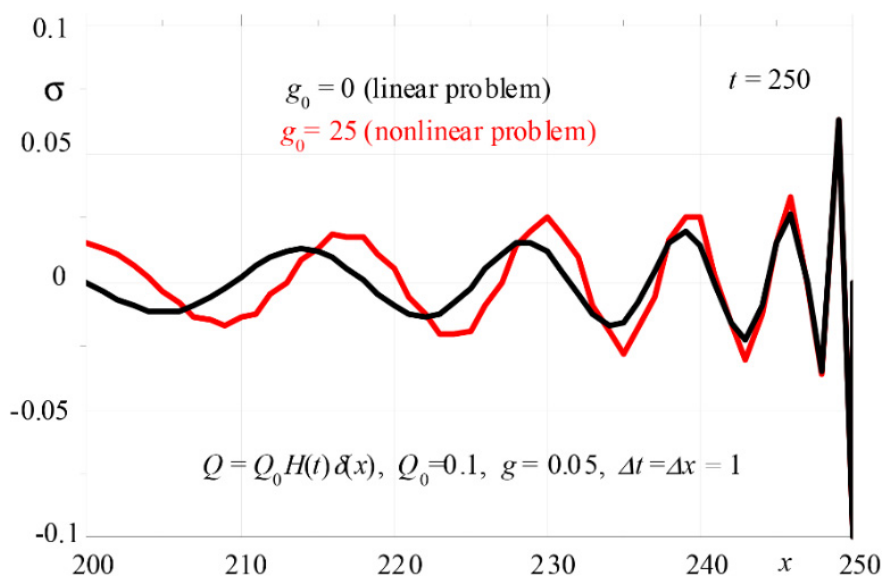

Figure 3: Snapshots (at $t=250$ ) of the strain distributions in linear and nonlinear problems.

\section{SPHERICAL AND CYLINDRICAL WAVES}

Consider the following wave equation possessing the inhomogeneous term:

$$
\ddot{u}=c_{0}^{2} u^{\prime \prime}+B(x) u^{\prime},
$$

where $B(x)$ is the finite function which can describe various models of continual media, for example, elastic waves in a thin rod of the variable the cross-section area or cylindrical and spherical waves in a compressible liquid under action of linear or point sources, respectively. In general, eqn (14) has no dispersion relation. As above, in Section 2, we apply the method of frozen coefficients (assume $|B(x)| \leq \bar{B} \sim$ const ) and then use the standard Fourier analysis to the difference analog of modified eqn (14). Then we obtained:

$$
u_{i}^{k+1}-2 u_{i}^{k}+u_{i}^{k-1}=\lambda^{2}\left(u_{i+1}^{k}-2 u_{i}^{k}+u_{i-1}^{k}\right)+\lambda \Delta t \bar{B}\left(u_{i+1}^{k}-u_{i-1}^{k}\right) / 2, \lambda=\Delta t / \Delta x .
$$

The resulting dispersion equation,

$$
c(q, \Delta x, \Delta t)=\frac{2}{q \Delta t} \arcsin \left[\lambda \sqrt{\sin ^{2} \frac{q \Delta x}{2}+\frac{\bar{B} \Delta x}{4} \sin (q \Delta x)}\right],
$$


shows that if to put $\lambda=1$ (remind, $c_{0}$ and $\Delta x$ are measurement units, then $\Delta t=1$ ), then. maximally short waves $(q=\pi)$ will propagate with phase velocity $c=1$. Now the MDM algorithm for calculation of eqn (15) with equality $\lambda=1$ is to be as follows:

$$
u_{i}^{k+1}=u_{i+1}^{k}+u_{i-1}^{k}-u_{i}^{k-1}+\bar{B}\left(u_{i+1}^{k}-u_{i-1}^{k}\right) / 2 .
$$

Consider cylindrical and spherical waves in a compressible liquid and set $B(x)=\alpha / x$. Then eqn (17) rewritten as

$$
\ddot{u}=u^{\prime \prime}+(\alpha / x) u^{\prime}, x>0
$$

will describe cylindrical $(\alpha=1)$ and spherical $(\alpha=2)$ pressure waves. Here $u$ plays role of the velocity potential, $x$ is the radial coordinate. In the wave, radial velocity and pressure are expressed as $\dot{u}=\partial u / \partial x$ and $P=-\partial u / \partial t$, respectively (the bulk compression modulus and the liquid density serve as measurement units). Under the condition $\lambda=1$, eqn (17) is turned out to the following $M D M$ algorithm (here $\Delta x=\Delta t=c_{0}=1$ ):

$$
u_{i}^{k+1}=u_{i+1}^{k}-u_{i}^{k-1}+u_{i-1}^{k}+\frac{\alpha}{2\left(x_{0}+i\right)}\left(u_{i+1}^{k}-u_{i-1}^{k}\right)
$$

where $x_{0}$ is the source radius, measured in steps $\Delta x$.

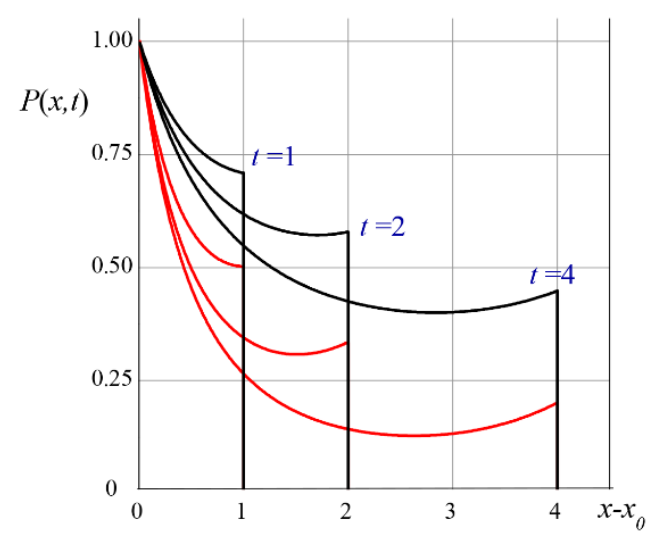

Figure 4: Pressure distribution at the moments of time $t=1,2,4$ in spherical (red lines) and cylindrical (black lines) waves under action of pressure $P(1, t)=H(t)$.

Let pressure of the Heaviside type, $P(1, t)=H(t)$, be set on cavity surfaces $\left(x_{0}=1\right)$ in both cases. In Fig. 4, calculated by $M D M$ algorithm (18), snapshots are shown of the pressure distributions along the radial coordinate at time values $t=1,2$ and 4 . The obtained numerical solutions completely agree with analytical ones (see, e.g. [18]). So, the ANSs are presented here.

Note that an analytical solution for the cylindrical case is not represented in a closed form and can be available as asymptotic one only in the small vicinity of the front. 


\section{FRACTURE DYNAMICS OF FIBER REINFORCED COMPOSITE PLATE}

The process of stresses concentration and stepwise crack propagation at fiber-adhesive interfaces of the fiber reinforced composite plate is numerically simulated. Note, some calculation results of delamination phenomena in layered structures were presented in [19], but the stepwise crack phenomena was not considered.

The problem statement corresponds to the following description: the material of the plate is stretched along the fibers at infinity by a constant tensile stress $\sigma_{\infty}$ (see Fig. 5). Here $E$ and $G$ are the Young module in fibers (black) and the shear module in adhesive (grey), $\rho$ and $\rho_{a}$ are densities, $h$ and $H$ are thicknesses, respectively. At zero time $(t=0)$, one of the fibers (say it be fiber $m=0$ ) starts to fail due to some defects.

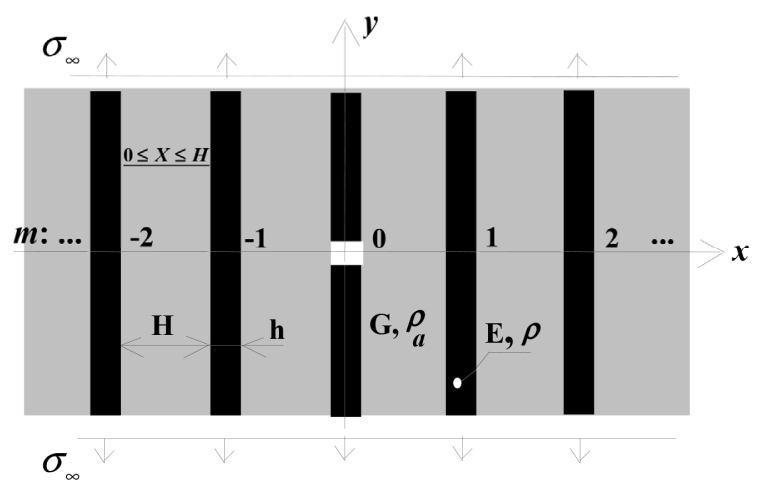

Figure 5: Loading of a unidirectional composite with the damage in a fiber.

Let $E$ and $G$ be the Young modulus in fibers and the shear module in adhesive respectively, $\rho$ and $\rho_{a}$ be densities, $h$ and $H$ be thicknesses of components as shown in Fig. 5, $c_{f}=\sqrt{E / \rho}$ and $c_{a}=\sqrt{G / \rho_{a}}$ be sound speeds in fibers and adhesive, respectively. Fiber constants $E, \rho, h$ serve as measurement units.

The fractured fiber starts to unload, and the intact ones start to load up due to action of shear stress waves propagating in the adhesive. Along with this process, the delamination events can happen depending on the strength of the adhesive.

The used model of the fiber dynamics describes the one-dimensional wave process in a thin rod embedded into the adhesive, which represented as inertial bonds perceived shear stresses (tension-compression stresses in adhesive bonds are neglected). Such a theoretical treatment of the components performance can be justified by the fact that the shear modulus of adhesive is much less than that of fiber (see e.g. [20]), while their stretches have roughly the same level due to the cohesion of fibers and the adhesive. When maximal stresses reached in adhesive do not exceed the strength limits $\left(\tau_{m}<\tau^{*}\right)$, the adhesive remains intact. The crack propagation is investigated on the basis of linear elastic fracture mechanics: fracture in adhesive is initiated if $\tau_{m} \geq \tau^{*}$, and then propagated deep into the composite up to their stop due to continuous scattering of the initial impact energy with time. 


\subsection{Mathematical formulation}

In the mathematical sense, we met a non-linear hyperbolic problem possessing non-classical boundary conditions. Due to the natural symmetry, a quarter of plane $x, y$ is considered in the calculation algorithm (let it be $x \geq 0, y \geq 0)$. At the initial state $(t<0)$ displacements and strains in fiber are

$$
u_{m}(y)=y \sigma_{\infty} / E, \text { or } \varepsilon_{m}(y)=\sigma_{\infty} / E ; v_{m}(X, y)=0(m=0, \pm 1, \pm 2, \ldots)
$$

where $\varepsilon_{m}(y)=\partial u_{m} / \partial y$ is the strain in $m$ th fiber $(m \neq 0)$, and the fracture event of fiber $m=$ 0 at $t=0$ changes eqn (19) by adding condition $\varepsilon_{0}(0, t)=0$ :

$$
\varepsilon_{0}(0)=0 ; \varepsilon_{m}(y)=\sigma_{\infty} / E(m \neq 0, y \neq 0), v_{m}(X, y)=0(m=0, \pm 1, \pm 2, \ldots)
$$

Then reformulate the problem for the additional dynamic state subtracting the static strains (19) from eqn (20). Then boundary conditions for strains in fibers are the following:

$$
y=0: \quad \varepsilon_{0}(0, t)=(\partial u / \partial y)_{m=0}=-\sigma_{\infty} / E, \varepsilon_{m}(0, t)=(\partial u / \partial y)_{m \neq 0}=0 .
$$

The motion of fibers is described by the system of $1 D$ wave equations

$$
\rho h \ddot{u}_{m}=E h u_{m, y}^{\prime \prime}+\tau_{m}^{+}(y)-\tau_{m}^{-}(y), m=0, \pm 1, \pm 2, \ldots,
$$

where $\tau_{m}^{+}$and $\tau_{m}^{-}$correspond to reactive shear forces at the fiber-adhesive interface on the right and the left, respectively:

$$
\tau_{0}^{+}=-\tau_{0}^{-}=\left.G v_{0, X}^{\prime}\right|_{X=0}, \tau_{m}^{+}=\left.G v_{m, X}^{\prime}\right|_{X=0}, \tau_{m}^{+}=\left.G v_{m-1, X}^{\prime}\right|_{X=H}(m>0),
$$

while displacements in adhesive described by wave equations

$$
\partial^{2} v_{m}(X, y, t) / \partial t^{2}=c_{a}^{2} \partial^{2} v_{m}(X, y, t) / \partial X^{2} \quad(0 \leq X \leq H), m=0,1,2, \ldots
$$

with boundary conditions:

$$
v_{m}(0, y, t)=u_{m}(y, t), \quad v_{m}(H, y, t)=u_{m+1}(y, t) .
$$

Then the following additional relations in expressions of reactive forces (23) and boundary conditions (25) are:

$$
\tau_{m}^{ \pm}\left(\xi^{ \pm}, y, t\right) \geq \tau^{*} \Rightarrow t_{m, \pm}^{*}=t ; t>t_{m, \pm}^{*}: \tau_{m}^{ \pm}\left(\xi^{ \pm}, y, t\right)=0, \partial v_{m}^{ \pm}\left(\xi^{ \pm}, y, t\right) / \partial X=0,
$$


where $\xi^{+}=0, \xi^{-}=H$, while indices “ \pm ” at $t_{m}^{*}$ denote right and left interfaces, respectively.

\subsection{The $M D M$ calculation algorithm}

It is evident that each possible scenario of wave-fracture pattern is saturated by reflected waves with discontinuities appeared due to adhesive cracking. Our goal is to calculate such processes as precisely as possible. Below we present the practical calculation device based on the $M D M$ technique allowing this goal to be reached. Let the mesh step in adhesive be $\Delta x$. Then the $M D M$ conditions are $\Delta t=\Delta y=1, \Delta x=c_{a}$, and the adhesive dynamics, as the analogue of eqn (24), described by the following $M D M$-algorithm:

$$
v_{m, j, i}^{k+1}=v_{m, j, i+1}^{k+1}+v_{m, j, i-1}^{k}-v_{m, j, i}^{k-1}(0 \leq i \leq s=H / \Delta x)
$$

while the $M D M$-algorithm of fibers dynamics in the difference analogue of eqn (22) can be written as

$$
\begin{aligned}
& u_{m, j}^{k+1}=u_{m, j+1}^{k}+u_{m, j-1}^{k}-u_{m, j}^{k-1}+\kappa F, F=\left(v_{m, j, i}^{k}+v_{m-1, j, i}^{k}\right)(m>0), F=2 v_{1, j, i}^{k}(m=0), \\
& \kappa=G H /(E h \mu), \mu=h \rho_{f} \Delta y+H \rho_{a} \Delta x .
\end{aligned}
$$

\subsection{Results of computer simulations}

In calculation examples below, parameters of composite are: $H=5, G=0.025, \rho_{a}=0.4$; stresses are normalized to $\sigma_{\infty}$, "critical values " $\sigma_{*}$ and " $\tau_{*}$ "are varied.

Let us turn to examples. The patterns of normal stresses $\sigma_{m}$ in fibers $m=1,2$ and 3 vs. time and shear stresses in interfaces $X=0$ and $X=H$ of the adhesive layer at $y=0$ are shown in Fig. 6.

It is convenient to relate stresses to their asymptotic values $\tau_{\mathrm{st}} \sim 0.053, \sigma_{\mathrm{st}}=4 / 3(t \rightarrow \infty)$ obtained in [21]. We note that peak amplitudes of shear stresses playing the main role in the fracture initiation process can be much more than $\tau_{\mathrm{s}}$. The peaks are appeared with the period $t=2 \mathrm{H} / \mathrm{c}_{a}$ due to reflections of shear waves, and their values do not change with time.
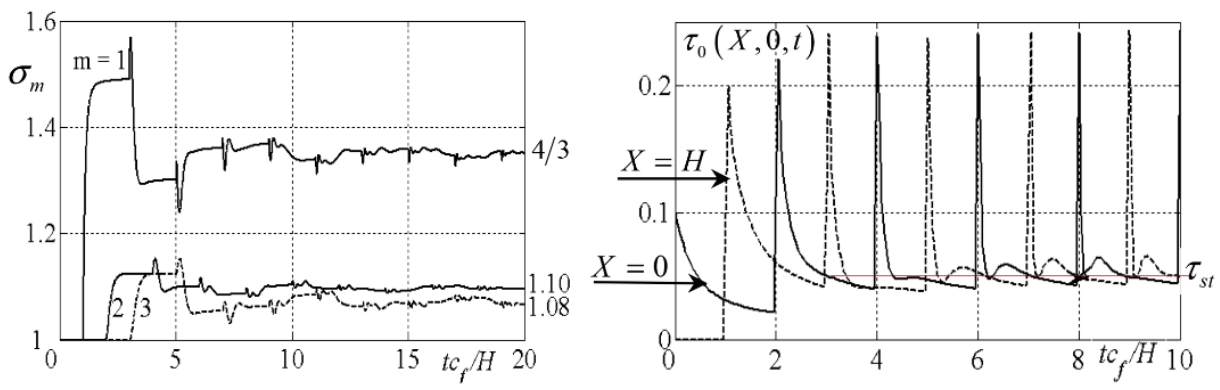

Figure 6: Normal stresses in fibers, $\sigma_{\mathrm{m}}$, and shear stresses in adhesive layer, $m=0$. 
The fracture propagation pattern in the case $\sigma^{*}=1.2$ and $\tau^{*}=0.1$ is shown in Fig. 7. After the initial rupture of 0th fiber $(t=0)$, the fracture in the adhesive occurs at the fiberadhesive interfaces $X=0$ (red), $X=H$ (blue) and possesses a high-speed avalanche-like pattern; the speed of fracture propagation decreases with time and the adhesive fracture is stopped at $t=30$ in interface $X=0$. Adhesive layers with $m \neq 0$ remain intact. Rapture of fibers occurs at $y=0$ and propagates with a little change in speed up to $t=63 \mathrm{H} / \mathrm{c}_{f}$, then it stopped.

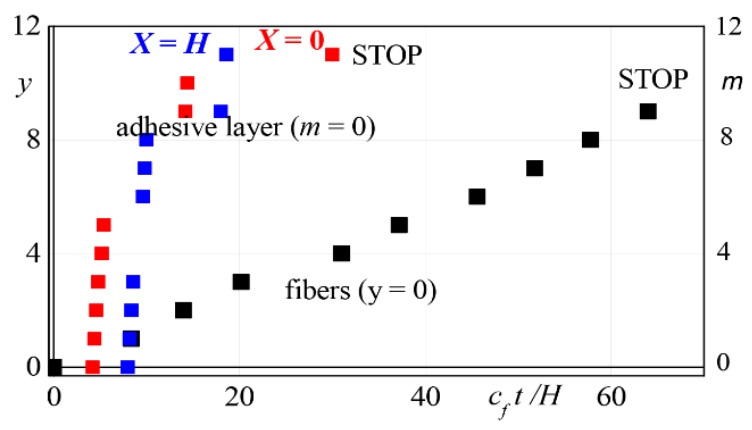

Figure 7: Fracture pattern in the composite vs. time $\left(\sigma^{*}=1.2, \tau^{*}=0.1\right)$.

Obtained results can be summarized in the main conclusions:

1. Designed $M D M$-algorithms allows high-gradient and step-wise character of wave processes to be precisely calculated.

2. The accurate $M D M$-solutions described front propagation patterns in media and structures are obtained for a set of linear and non-linear $1 D$ transient wave problems.

3. The $M D M$-algorithms built for exploring the $2 D$ processes of stress concentrations and progressive fracture of fiber reinforced composite plates allow one to analyze fracture development heavily caused by discontinuities of shear waves propagated in the adhesive.

\section{REFERENCES}

[1] Weinberger, H.F., Upper and lower bounds for eigenvalues by finite difference methods. Communications on Pure and Applied Mathematics, 9, pp. 613-623, 1956.

[2] Lax, P.D. \& Wendroff, B., Difference schemes for hyperbolic equations with high order of accuracy. Communications on Pure and Applied Mathematics, 17, pp. 381392, 1964.

[3] Fromm, J.E., A method for reducing dispersion in convective difference schemes. Journal of Computational Physics, 3, pp. 176-183, 1968.

[4] Chin, R.C.Y., Dispersion and Gibbs phenomenon associated with difference approximations to initial boundary-value problems for hyperbolic equations. Journal of Computational Physics, 18(3), pp. 233-247, 1975.

[5] Abdukadirov, S.A., Pinchukova, N.I. \& Stepanenko, M.V., A numerical solving dynamic equations of elastic media and structures. Journal of Mining Science, $\mathbf{6}$, pp. 19-22, 1984. 
[6] Belov, A.I., Kornilo, V.A. \& Stepanenko, M.V., Reaction of a three-layer hydroelastic cylindrical shell on the effect of axisymmetric internal explosion. Journal of Applied Mechanics and Technical Physics, 1, pp. 49-57, 1986.

[7] Abdukadirov, S.A., Kurmanaliev, K.K. \& Stepanenko, M.V., Dynamic stresses on the perimeter of a rigid inclusions embedded in a rock bed. Journal of Mining Science, 24, pp. 442-447, 1988.

[8] Abdukadirov, S.A., Alexandrova, N.I. \& Stepanenko, M.V., Non-steady diffraction of a plane longitudinal wave on an elastic cylindrical shell. Mechanics of Solids, 5, pp. 132-137, 1989.

[9] Slepyan, L.I. \& Ayzenberg-Stepanenko, M.V., Penetration of metal-fabric composite targets by small projectiles. Personal Armor Systems. British Crown Copyright/MOD: Colchester, UK, 1998.

[10] Ayzenberg-Stepanenko, M.V.\& Slepyan, L.I., Localization of strain and melting wave in high-speed penetration. IUTAM Symposium Nonlinear Singularities, Kluwer, 1999.

[11] Kubenko, V.D. \& Ayzenberg-Stepanenko, M.V., Impact indentation of a rigid body into elastic layer. Journal of Mathematical Sciences, 1, pp. 156-171, 2009.

[12] Int-Veen, R., Avoiding numerical dispersion in option valuation. Computer Visual Science, 10(4), pp. 556-564, 2007.

[13] Wu, Y.S. \& Forsyth, P.A., Efficient schemes for reducing numerical dispersion in modeling multi-phase transport through heterogeneous geological media. Vadose Zone Journal, 7(1), pp. 340-349, 2008.

[14] Sun, Z., Ren, Y., Larricq, C., Zhang, S. \& Yang, Y., A class of finite difference schemes with low dispersion and controllable dissipation for DNS of compressible turbulence. Journal of Computational Physics, 230(12), pp. 231-244, 2011.

[15] Zhou, Y., Yang, D., Ma, X. \& Li, J., An effective method to suppress numerical dispersion in 2D acoustic and elastic modeling using a high-order Padé approximation. Journal of Geophysics and Engineering, 12(1), pp. 114-129, 2015.

[16] Wu, Z. \& Alkhalifah, T.A., Highly accurate finite-difference method with minimum dispersion error for solving the Helmholtz equation. Journal of Computational Physics, 365, pp. 350-361, 2018.

[17] Cheng, Y., Guangzhi, C., Xiang-Hua, W. \& Shunchuan, Y., Investigation of numerical dispersion with time step of the FDTD methods: avoiding erroneous conclusions. IET Microwaves, Antennas and Propagation, pp. 1-13, 16 Apr. 2021.

DOI: $10.1049 / \mathrm{mia} 2.12068$.

[18] Slepyan, L.I., Nonstationary Elastic Waves, Sudostrojenie: Leningrad, 1972. (In Russian.)

[19] Nayak, A.K., Shenoi, R.A. \& Moy, S.J., Transient response of initially stressed composite plates. Finite Elements in Analysis and Design, 2(10), pp. 821-836, 2006.

[20] Summerscales, J. ed., Microstructural Characterisation of Fibre-Reinforced Composites, Woodhead Publishing: Cambridge, 1998.

[21] Michailov, A.M., Dynamics of a unidirectional fiberglass. Journal of Applied Mechanics and Technical Physics, 4, pp. 139-145, 1974. 